\title{
"Caracterización fisicoquímica, funcional y reológica de harina de cáscara de maracuyá (Passiflora edulis SIMS)"
}

\section{Physicochemical, functional and rheological characterization of passion fruit shell flour (Passiflora edulis SIMS)}

\author{
Saúl Ricardo Chuqui-Diestra ${ }^{1}$ y Luz María Paucar-Menacho ${ }^{2}$ \\ ${ }^{1}$ Universidad Nacional de San Cristóbal de Huamanga, Perú. \\ ${ }^{2}$ Universidad Nacional del Santa, Perú.
}

\begin{abstract}
RESUMEN
La cáscara de maracuyá es un residuo agroindustrial que con valor agregado se puede utilizar en la industria alimentaria. Este trabajo tuvo por finalidad la caracterización fisicoquímica, funcional y reológica de la harina de cáscara de maracuyá (HCMr). Se realizaron análisis fisicoquímicos, funcionales y reológicos. El valor de humedad de HCMr fue de 11,25\%, esta se encuentra dentro de las exigencias de las normas peruanas. Los valores de proteína $(5,14 \%)$ son bajos comparados con harinas de cerelaes. El valor de fibra dietética total fue de $63,88 \%$. Los valores totales de polifenoles y actividad antioxidante fueron 504,75 mg G.A.E./100g y 1520,49 $\mu \mathrm{mol}$ ET/100g respectivamente. El valor de capacidad de retención de agua ascendió a $12,93 \mathrm{ml}$ agua retenida/g. Los valores reológicos fueron: 702 unidades farinográficas de consistencia con 14,02\% de humedad. Con estas características obtenidas la HCMr puede utilizarse como ingrediente o sustituto alimentario y así obtener productos con bondades nutritivas importantes.
\end{abstract}

Palabras clave: compuestos bioactivos, fibra dietaria, propiedades reológicas, capacidad de retención de agua.

\begin{abstract}
Passion fruit peel is an agro-industrial waste that with added value can be used in the food industry. The purpose of this work was the physicochemical, functional and rheological characterization of passion fruit shell flour (HCMr). Physicochemical, functional and rheological analyzes were carried out. The humidity value of $\mathrm{HCMr}$ was $11.25 \%$, this is within the requirements of Peruvian standards. Protein values (5.14\%) are low compared to cerelae flours. The total dietary fiber value was $63.88 \%$. The total values of polyphenols and antioxidant activity were $504.75 \mathrm{mg}$ G.A.E./100g and 1520.49 $\mu \mathrm{mol}$ ET / 100g respectively. The water retention capacity value was $12.93 \mathrm{ml}$ retained water / g. The rheological values were: 702 consistency farinographic units with $14.02 \%$ humidity. With these characteristics obtained, HCMr can be used as an ingredient or food substitute and thus obtain products with important nutritional benefits.
\end{abstract}

Keywords: bioactive compounds, dietary fiber, rheological properties, water retention capacity.

Saúl Ricardo Chuqui-Diestra. ORCID: https://orcid.org/0000-0003-2582-2716 email: saul.chuqui@ unsch.edu.pe Luz María Paucar-Menacho. ORCID: https://orcid.org/0000-0001-5349-6167 email: luzpaucar@uns.edu.pe 


\section{INTRODUCCIÓN}

$\mathrm{E}^{\mathrm{n}}$ n la agroindustria se generan subproductos propios del procesamiento de productos hortofrutícolas, estos residuos se pueden utilizar en diversas alternativas, por la composición tan variada que presentan, entre los más importantes y relevantes están las cáscaras, semillas, restos de pulpas, entre otros. (Gustavsson et al., 2013).

Diversos estudios demostraron que la harina de cáscara de maracuyá disminuyó los índices de colesterol en damas con edades que fluctuaron entre 30 a 60 años que presentaban altos valores ( $\geq 200 \mathrm{mg} / \mathrm{dL}$ ), la cáscara de esta fruta contiene valores altos de niacina (vitamina B3), calcio, hierro y fósforo. (Quintero, 2013).

Cruz et al., (2015) refieren que la cáscara de maracuyá tiene un $55,42 \%$ de fibra dietaria total, siendo el $41,19 \%$ fibra dietaria insoluble y el 9,04\% es fibra dietaria soluble; la fibra dietaria insoluble está conformada en: hemicelulosa A $(2,35 \%)$, hemicelulosa B $(4,47 \%)$, celulosa $(15 \%)$ y lignina $(21,49 \%)$.

La harina de cáscara de passiflora mayormente contiene pectina de valioso metóxilo que tiene beneficios para las personas; actualmente coadyuvan a reducir colesterol en la sangre y niveles de azúcar, y es rica compuestos fenólicos. (Calderón y Noriega, 2017).

La relevancia que tiene el consumo de fibra dietaria (FD) ha conllevado a desarrollar nuevos productos a la industria alimentaria, que sean más saludables y que presenten contenidos importantes de fibra dietaria, vitaminas y bajos contenidos de colesterol (Saura-Calixto y Jiménez-Escrig, 2001 citado por Ivana, 2013).

Presta una mayor importancia la capacidad de retención de agua que presenta la fibra dietaria, en base a la formulación y transformación alimenticia ricos en fibra, debido a que esta característica depende fundamentalmente del nivel máximo de sustitución de la misma (Ruiz, 2016).

Terpinc et al., (2012) precisan que el color, aspectos organolépticos (sabor, astringencia, textura), propiedades nutritivas y antioxidantes de los alimentos hortofrutícolas están asociadas a los compuestos fenólicos.

La estabilidad de emulsiones en productos con contenidos elevados de grasa es favorecida por la alta capacidad de adsorción de aceite (Elleuch et al., 2011).

Decker (1997) precisa que la capacidad antioxidante de las sustancias fenólicas se relaciona con su propiedad de secuestrar metales, inactivar la enzima lipoxigenasa y captar radicales libres, en algunos casos es posible generar reacciones de oxidación "in vitro".

La farinografía determina la resistencia de la mezcla al amasado y consiste en evaluar en base a prueba y posible error, los resultados óptimos de agua para obtener la consistencia ideal de la masa (500 Unidades Brabender) (Serna, 2013).

Grass et al., (2000) refieren que la tolerancia a la masa, la maquinabilidad, tolerancia fermentativa y que los valores reológicos se puedan mantener en lo posible constantes son características muy importantes de una harina para el proceso de panificación.

Esta investigación tuvo por propósito la caracterización fisicoquímica, funcional y reológica de la harina de cáscara de maracuyá (Passiflora edulis SIMS) a finde obtener un producto con cualidades nutritivas y funcionales y pueda ser usado como ingrediente o sustituto en la industria alimenticia.

\section{Materiales y Métodos}

\section{Materia prima, insumos y lugar de ejecución}

Para obtener harina de cáscara de maracuyá (HCMr) se realizó en las instalaciones del Instituto de Investigación Tecnológica Agroindustrial (IITA) de la Universidad Nacional del Santa (UNS), se utilizó cáscara de maracuyá procedente de la Empresa Delicias y Sabores E.I.R.L. ubicado en la ciudad de Casma, Región Ancash. Los ensayos experimentales fueron realizados en el IITA y Laboratorio de Composición de Productos Agroindustriales de la UNS.

\section{Obtención de harina de cáscara de maracuyá}

Se optaron por aquellas cáscaras de maracuyá libres de deterioro. Se recolectó en el laboratorio, para luego lavar con abundante agua corriente y se desinfectó con hipoclorito de sodio ( $5 \mathrm{ml} / 10$ lt de agua), después se cortó manualmente en trozos homogéneos de $3 \times 3 \mathrm{~cm}$, se escaldó a ebullición por 4 minutos para inactivar enzimas responsables del pardeamiento enzimático.

En el deshidratador de bandejas se procedió a deshidratarlas a $70^{\circ} \mathrm{C}$, con flujo de aire caliente de $15 \mathrm{~m} / \mathrm{s}$ por 8 horas, luego se efectuó el proceso de molienda en un molino convencional y finalmente se tamizaron con un juego de tamices $\mathrm{N}^{\circ} 16,20,30$ y $40(380 \mu)$ para obtener una harina homogénea, con una granulometría de $250 \mu \mathrm{m}$.

En la figura 1 se observa el flujograma de la obtención de harina a base de cáscara de maracuyá.

\section{Evaluación fisicoquímica de la harina a base de cáscara de maracuyá}

a) Humedad: Por diferencia de pesos, se determinó por triplicado. (AOAC, 2006)

b) Proteínas: Método Kjeldahl, método 928.08. (AOAC, 2006).

c) Grasa: Método Soxhlet con el extractor FOSS tipo SOXTEC, usando éter de petróleo como solvente, método 963.15. (AOAC, 2006).

d) Cenizas: Por incineración directa, método 923.03 . (AOAC, 2006).

\section{e) Análisis de granulomtría}

Para evaluar la granulometría de HCMr se utilizó el equipo RETSCH. Los tamices pesados previamente, se pasaron $50 \mathrm{~g}$ de harina por 15 minutos en 5 tamices redondos cuyas mallas fueron de $28,42,60,80,115,170$ y 200 tipo Tyler y lo retenido en cada tamiz se pesaron y se expresaron en porcentaje. Método citado en Moreno (2017). 
ISSNe: 2617-9156

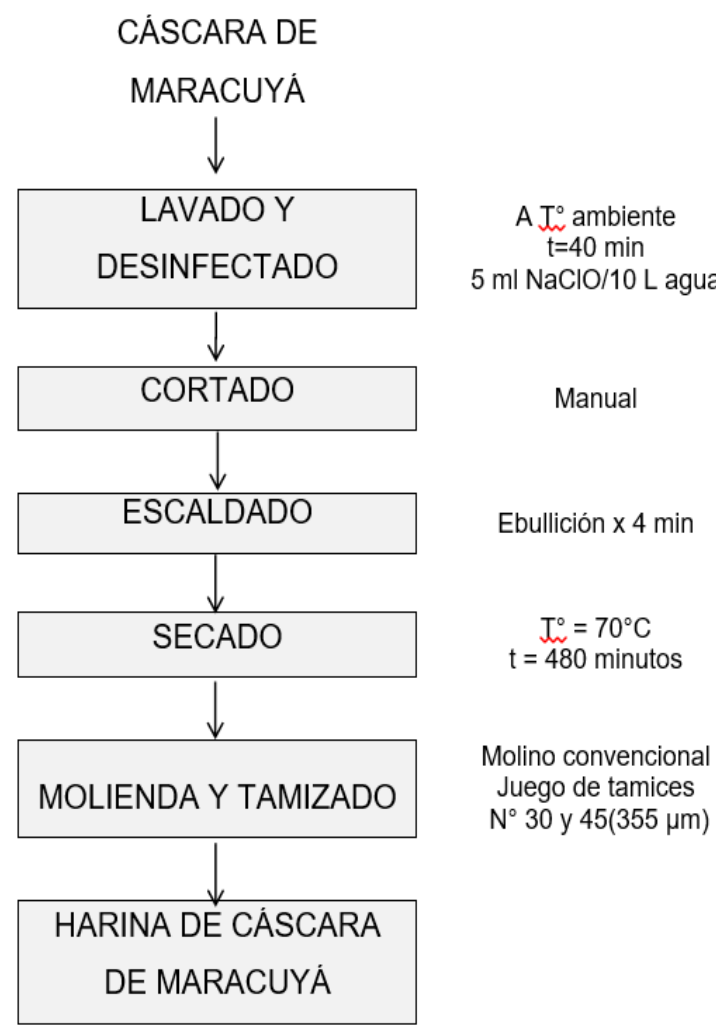

Figura 1:

Diagrama de flujo para obtener harina de cáscara de maracuyá.

Evaluación fisicoquímica de la harina a base de cáscara de maracuyá.

a) Capacidad de retención de agua (CRA): En un tubo para centrífuga pesado previamente se colocó 1 gramo de HCMr, se dispersó en $10 \mathrm{~mL}$ de agua destilada con agitación constante por $1 \mathrm{~min}$, las muestras reposaron por 30 min y se centrifugaron a 3000 RPM por 30 minutos en probetas de $10 \mathrm{~mL}$, se recuperó el agua liberada en la centrifugación. La CRA fueron expresados en $\mathrm{mL}$ de agua retenida por $\mathrm{g}$ de muestra. Método recomendado por Polanco (2017).

b) Capacidad de adsorción de aceite (CAA): En un tubo de ensayo se pesó 1 gramo de HCMr, se agregó $5 \mathrm{~mL}$ de aceite y se homogenizó por 30 minutos; se dejó reposar por $24 \mathrm{~h}$ en temperatura medioambiental, para luego centrifugar a 3000 RPM por espacio de 10 minutos, se separó el sobrenadante para luego pesar lo sedimentado (g). Método citado en Miguel (2008).

c) Fibra dietaria total (FDT), insoluble (FDI) y soluble (FDS): La metodología empleada para determinar FDT, FDI y FDS se basó en la metodología AOAC 991.43 "Fibra Dietética Total, Soluble e Insoluble en Alimentos" y AACC 3207 "Determinación de Fibra Dietética Soluble, Insoluble y Total en Alimentos y Productos Alimenticios". Es simplificado del método AACC 32-05 "Fibra Dietética Total", y de AACC 32-21 "Fibra Dietética Soluble e Insoluble para productos de avena" (Megazyme Manual, 2011). d) Contenido total de polifenoles: Se utilizó el método de Folin-Ciocalteu descrito por Singleton y Rossi (1965) citado en Jurado et al., (2016).

e) Capacidad antioxidante (DPPH): La actividad antioxidante de los extractos se evaluaron por el principio de capacidad receptora del radical DPPH empleando el método de Rojano et al., (2008) citado en Palomino et al., (2009).

Análisis reológico de harina a base de cáscara de maracuyá.

La caracterización reológica de $\mathrm{HCMr}$ se fundamentó en el análisis farinográfico.

\section{a) Farinografía}

En el Farinógrafo Brabender se evaluaron la capacidad de absorción de agua y características de la mezcla de harina (AACC, 1995). La absorción, tiempo óptimo de desarrollo, estabilidad, índice de tolerancia y tiempo de caída fueron parámetros evaluados por el farinógrama.

\section{DISCUSIÓN}

Caracterización fisicoquímica de la harina de cáscara de maracuyá.

a) Rendimiento

En el procesamiento de maracuyá en la Empresa Delicias y Sabores E.I.R.L., el rendimiento de pulpa es de $31-32 \%$ y de cáscara $50-51 \%$, en un turno de 8 horas se procesan $20-25 \mathrm{TM}$ de fruta obteniéndose aproximadamente $12000 \mathrm{~kg}$ de cáscara por día.

En la tabla 1 se detalla el rendimiento de cáscara de maracuyá, deduciéndose que a partir de $50 \mathrm{~kg}$ de cáscara de maracuyá, se logró obtener 4,64 kg de cáscara deshidratada y $4,49 \mathrm{~kg}$ de harina tamizada con granulometría adecuada. El rendimiento de harina en función de cascara fresca fue de 8,98\%; valores de 13,7\% reportaron Chung et al (2019) de la variedad amarillo. Estas diferencias pueden deberse al lugar de cultivo, condiciones de secado, grosor y tamaño de la cáscara, entre otros.

Tabla 1

Rendimiento de harina de cáscara de maracuyá.

\begin{tabular}{lcc}
\hline Componente & Porcentaje (\%) & Peso $(\mathbf{K g})$ \\
\hline $\mathrm{CMr}$ & 51 & 50 \\
CMr deshidratada & 9,28 & 4,64 \\
HCMr molida & 98,71 & 4,58 \\
HCMr tamizada & 98,06 & 4,49 \\
\hline
\end{tabular}

CMr: cáscara de maracuyá.

\section{b) Análisis proximal}

La tabla 2 muestra el análisis proximal de HCMr. Los valores de humedad fueron de $11,25 \%$, valores ligeramente mayores $(12,04 \%)$ y $(12,17 \%)$ reportaron Arteaga y Silva (2015) y Villanueva (2018) respectivamente; encontrándose en el rango permisible para harinas sustitutas de trigo (15\%) descritas en NTP 205.040: 2016.

Los valores de proteína $(5,14 \%)$, grasa $(0,6 \%)$, cenizas 
ISSNe: $2617-9156$

$(4,93 \%)$ y fibra $(28,33 \%)$ fueron ligeramente mayores a lo reportado por Villanueva (2018). Estas variaciones se deben a condiciones de cultivo, variedad, estado de madurez, lugar de cultivo y fechas cosechadas.
En el análisis proximal de $\mathrm{HCMr}$ resaltan los valores de fibra bruta, característica particular que le confiere a este producto.

\section{Tabla 2}

Composición proximal de la harina de cáscara de maracuyá.

\begin{tabular}{cc}
\hline Componente & Valores $\mathbf{( \% )}$ \\
\hline Humedad $^{1}$ & $11,25 \pm 0,04$ \\
Proteína $^{1}$ & $5,14 \pm 0,01$ \\
Grasa $^{1}$ & $0,60 \pm 0,02$ \\
Cenizas $^{1}$ & $4,93 \pm 0,02$ \\
Carbohidratos & 49,78 \\
Fibra bruta $^{1}$ & $28,33 \pm 0,29$ \\
\hline
\end{tabular}

Media de 3 repeticiones $\pm \overline{\mathrm{DS}}$

c) Análisis granulométrico

La tabla 3 presenta la distribución granulométrica de HCMr. En la Normas CODEX STAN 152 (1995) refiere que la granulometría de la harina de trigo debe pasar a través de un tamiz de $\mathrm{N}^{\circ} 70(212 \mu \mathrm{m})$ en un $98 \%$. En el tamizado de HCMr la mejor retención fue en del tamiz $\mathrm{N}^{\circ}$ $60(250 \mu \mathrm{m})$, esto se corrobora en la figura 2. Según
Fuertes (1998) citado por Paucar (2014) precisa que el tamaño de partícula adecuado para harinas debe ser de 50 a $500 \mu \mathrm{m}$.

Al utilizar harina de los tamices de $250 \mu \mathrm{m}$ y debajo de ellas, puede considerarse adecuado para su sustitución en productos alimenticios.

\section{Tabla 3}

Distribución granulométrica de la harina de cáscara de maracuyá.

\begin{tabular}{cccc}
\hline Número de tamiz (TYLER) & Tamaño de partícula $(\boldsymbol{\mu m})$ & Cantidad retenida $(\mathbf{g})$ & Porcentaje de retención $(\%)$ \\
\hline 28 & 600 & $0,38 \pm 0,01$ & $0,76 \pm 0,05$ \\
42 & 355 & $4,23 \pm 0,09$ & $3,48 \pm 0,11$ \\
60 & 250 & $12,43 \pm 0,15$ & $74,91 \pm 0,18$ \\
80 & 180 & $9,12 \pm 0,11$ & $8,28 \pm 0,09$ \\
115 & 125 & $6,13 \pm 0,08$ & $2,29 \pm 0,07$ \\
170 & 90 & $5,16 \pm 0,02$ & $0,34 \pm 0,01$ \\
200 & 75 & $2,56 \pm 0,03$ & $0,13 \pm 0,02$ \\
Base o plato & - & $9,88 \pm 0,15$ & $9,80 \pm 0,16$ \\
\hline
\end{tabular}

Media de 3 repeticiones \pm DS

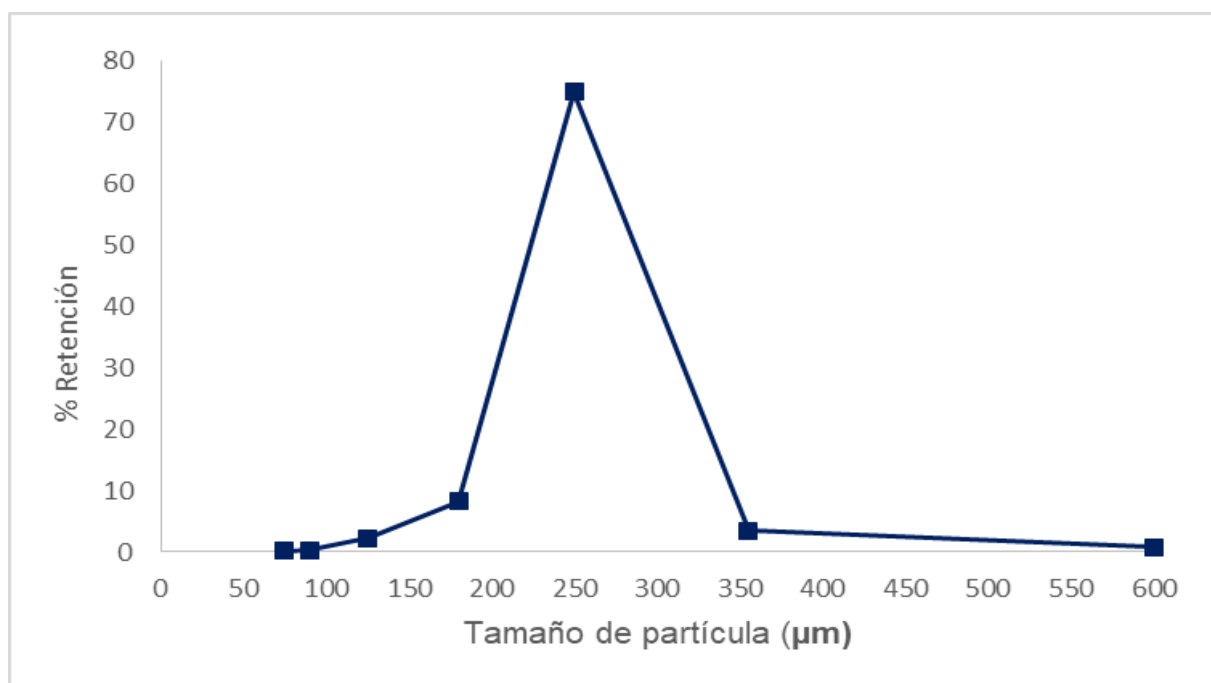

Figura 2:

Valores de \% de retención de HCMr en función del tamaño de partícula 
ISSNe: 2617-9156

Caracterización fisicoquímica de la harina de cáscara de maracuyá.

a) Capacidad de retención de agua (CRA)

La tabla 4 muestra los valores de CRA de HCMr. Se observa que la $\mathrm{HCMr}$ del tamiz $\mathrm{N}^{\circ} 60(250 \mu \mathrm{m})$ es el más recomendable por tener valor mayor de CRA frente al tamiz $\mathrm{N}^{\circ} 80(180 \mu \mathrm{m})$.

Leterme et al., (1998) citado Núñez (2014) refieren que la fibra de frutas presentan una CRA mayor a 10 gramos de agua/g de harina. Abarca (2010) precisa que a mayor fracción soluble en fibra de frutas mayor será la CRA.

Tabla 4

Valores de CRA de harina de cáscara de maracuyá.

\begin{tabular}{ccc}
\hline $\begin{array}{c}\text { Número de } \\
\text { tamiz } \\
\text { (TYLER) }\end{array}$ & $\begin{array}{c}\text { Tamaño de } \\
\text { partícula } \\
(\boldsymbol{\mu m})\end{array}$ & $\begin{array}{c}\text { CRA* }(\mathbf{m L} \\
\text { agua } \\
\text { retenida/g } \\
\text { muestra) }\end{array}$ \\
\hline 60 & 250 & $12,93 \pm 0,15$ \\
80 & 180 & $11,81 \pm 0,11$ \\
\hline
\end{tabular}

* Media de 3 repeticiones \pm DS

b) Capacidad de adsorción de aceite (CAAc)

En la tabla 5 se muestra los valores de capacidad de retención de aceite de la harina de cáscara de maracuyá. Los valores de CAAc encontrados en este estudio para tamices $\mathrm{N}^{\circ} 60(250 \mu \mathrm{m})$ y $\mathrm{N}^{\circ} 80(180 \mu \mathrm{m})$, estuvieron entre 2,66 y 2,89 $\mathrm{g}$ aceite/g harina respectivamente, valor ligeramente superior a lo reportado por Matsuura (2005) que fue de 2,5 $\mathrm{g}$ aceite/g harina.

Schweizer (1992) citado en Núñez (2014) precisa que la capacidad de adsorción de aceite en harinas de residuos hortofrutícolas son menores a $2 \mathrm{~g}$ aceite/g de harina y para cereales los valores fluctúan entre 2 a $4 \mathrm{~g}$ aceite/g harina.

Tabla 5

Valores de CAAc de harina de cáscara de maracuyá.

\begin{tabular}{ccc}
\hline $\begin{array}{c}\text { Número de } \\
\text { tamiz } \\
\text { (TYLER) }\end{array}$ & $\begin{array}{c}\text { Tamaño } \\
\text { de } \\
\text { partícula } \\
(\boldsymbol{\mu m})\end{array}$ & $\begin{array}{c}\text { CAAc* } \\
(\mathbf{m L} \text { aceite } \\
\text { retenido/g muestra) }\end{array}$ \\
\hline 60 & 250 & $2,89 \pm 0,02$ \\
80 & 180 & $2,66 \pm 0,01$ \\
\hline
\end{tabular}

* Media de 3 repeticiones \pm DS

c) Fibra dietética total (FDT), fibra dietética insoluble (FDI) y fibra dietética soluble (FDS)

En la tabla 6, se muestra los valores obtenidos de FDT, FDI y FDS representando valores muy importantes en HCMr y que su consumo en los productos elaborados sustitutos contribuye a la salud humana. Cruz et al., (2015) reportaron valores de FDT $(52,42 \%)$, FDI $(41,19 \%)$ y FDS $(9,04 \%)$. Escudero y González (2006) recomiendan en adultos el consumo entre 20-35 g/día de fibra dietética, o 10-14 g por cada $1000 \mathrm{kcal}$; y que en el consumo de fibra debería tener una relación de 3/1 entre insoluble y soluble.

La fibra dietaria insoluble es recomendable porque mejoraran el volumen y a la vez mantiene frescos los productos de panificación por su capacidad de retener agua (Cruz et al., 2015).

\section{Tabla 6}

Valores de FDT, FDI y FDS de harina de cáscara de maracuyá.

\begin{tabular}{cc}
\hline Componente & Valores (\%) \\
\hline FDT & $63,88 \pm 1,09$ \\
FDI & $49,53 \pm 1,21$ \\
FDS & $14,76 \pm 0,14$ \\
\hline
\end{tabular}

Media de 3 repeticiones \pm DS

d) Polifenoles totales y actividad antioxidante

En la tabla 7 se muestra los valores de polifenoles y actividad antioxidante de HCMr que fueron 504,22 mg G.A.E./100 g y 1520,49 $\mu \mathrm{mol}$ ET/100g respectivamente, valores de polifenoles totales inferiores reportaron Caballero y Escobedo (2019) a temperatura de secado de $50^{\circ} \mathrm{C}$, de $95 \mathrm{mg}$ A.G./100 g y valores muy altos de capacidad antioxidante de 9337,56 $\mu \mathrm{mol}$ ET/100 g. Estas diferencias se pueden deber a la variedad del fruto, suelo, prácticas de cultivo, entre otros.

Tabla 7

Valores de polifenoles y actividad antioxidante de HCMr.

\begin{tabular}{lc}
\hline \multicolumn{1}{c}{ Componente } & \multicolumn{1}{c}{ Valores } \\
\hline Polifenoles totales $(\mathrm{mg}$ & $504,75 \pm 1,20$ \\
G.A.E./100 g) & $1520.49 \pm 1,54$ \\
Actividad antioxidante & \\
$(\mu$ mol ET/100 g) & \\
\hline Media de 3 repeticiones \pm DS &
\end{tabular}

\section{Caracterización reológica de harina de cáscara de maracuyá.}

La HCMr no contiene gluten, esta compuesto por fibra con alto contenido de pectinas de valioso metóxilo, también contiene niacina, hierro, calcio, fósforo y compuestos fenólicos (Calderón y Noriega, 2017).

Para determinar las características reologícas de HCMr se realizó una mezcla con agua.

\section{Farinografía}

En la tabla 8 se presenta los valores farinográficos de la HCMr.

\section{Tabla 8}

Parámetros farinográficos de HCMr.

\begin{tabular}{lc}
\hline \multicolumn{1}{c}{ Parámetro } & HCMr \\
\hline Humedad (\%) & $14,02 \pm 0,06$ \\
Absorción (\%) & $74,3 \pm 1,22$ \\
Tiempo óptimo de desarrollo (min) & $1,2 \pm 0,01$ \\
Estabilidad (min) & $0,3 \pm 0,01$ \\
Índice de tolerancia (FU) & $158 \pm 1,55$ \\
Tiempo de caída (min) & $1,5 \pm 0,01$
\end{tabular}

Media de 3 repeticiones \pm DS 
En la figura 3 se presenta el perfil farinográfico de HCMr.

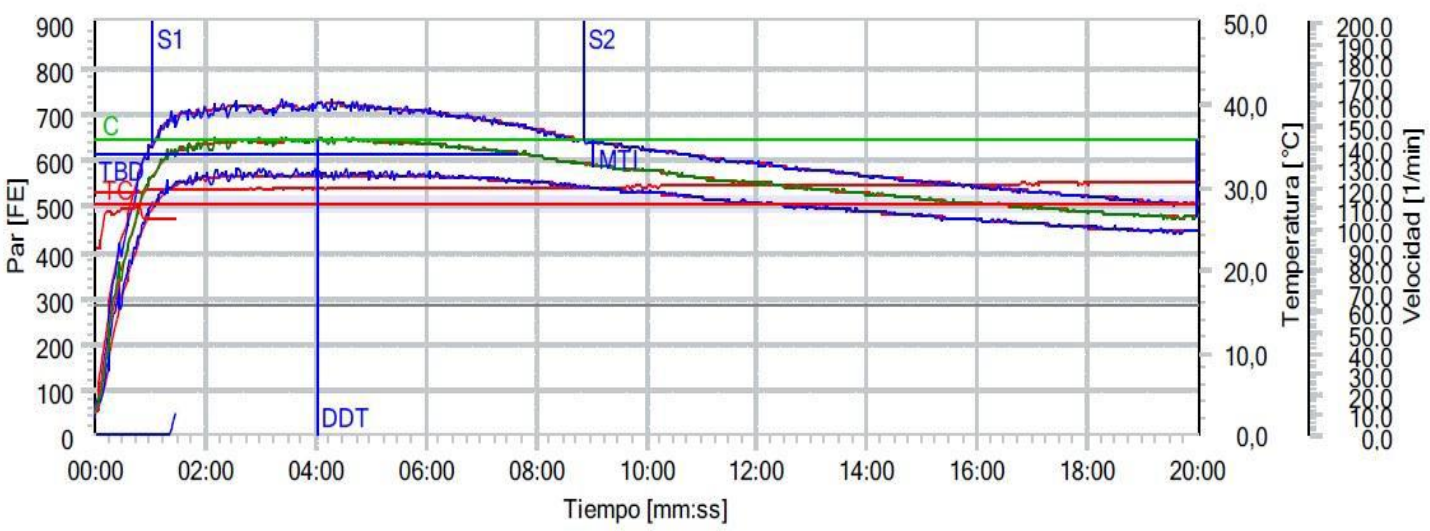

Fuente: FARINOGRAFO-E de Brabender®.

Laboratorio de Análisis y Composición de productos agroindustriales - UNS

Figura 3:

Perfil farinográfico de $\mathrm{HCMr}$

Al observar la figura 3 y tabla 8 se puede deducir que para la mezcla de HCMr el índice de tolerancia al mezclado se encuentra por debajo del valor de $500 \mathrm{UF}$, cuyo valor de absorción fue de 74,3\%; valores cercanos reportó Chávez (2014) en harina de quequi $(73,6 \%)$ y harina para pan de barra $(77,6 \%)$.

Chávez (2011) precisa que harinas sin gluten presentan valores de absorción característica de una harina fuerte pero son inestables y no toleran adecuadamente el amasado por tener valores superiores a 120 UF.

Cazares (2011) refiere que los porcentajes de absorción de las harinas durante el amasado está en función de la granulometría de la harina, el contenido proteico y de almidón, el valor inicial de humedad y la humedad relativa del entorno

\section{COnClusiones}

El rendimiento de $\mathrm{HCMr}$ fue de $8,98 \%$, este valor refleja la importancia del valor agregado de este subproducto.

Los valores de humedad, proteína, grasa, cenizas de la HCMr lo enmarcan dentro de los límites normativos para una harina, y los altos contenidos de fibra le confieren un producto con cualidades nutricionales importantes.

El análisis granulométrico de HCMr lo ubican dentro de las normas para ser utilizado como sustitutos de harinas.

La buena CRA, CAAc, alta proporción de fibra dietética, contenido importante de polifenoles, buena capacidad antioxidante y valores farinográficos importantes le confieren a la $\mathrm{HCMr}$ características especiales para poder ser utilizada como ingrediente o sustituto en la industria alimenticia a fin de aportar cualidades fisicoquímicas y funcionales al producto final.

\section{REFERENCIAS}

[1] Abarca, D. (2010). Identificación de fibra dietaria en residuos de cacao (Theobroma cacao L.) variedad complejo nacional por trinitario. (Título profesional, Universidad Técnica Particular de Loja). Repositorio Institucional

UPTL. http://dspace.utpl.edu.ec/handle/123456789/13014.

[2] AOAC, 2006. Official Methods of Analysis of AOAC International, 18th ed. AOAC International, Maryland, USA.

[3] Arteaga P y Silva A. (2015). Sustitución parcial de la harina de trigo (Triticum aestivum) por harina de tarwi (Lupinus mutabillis SWEET) y harina de cascara de maracuyá (Passiflora edulis) en las características fisicoquímicas y sensoriales de cupcakes. (Título profesional, Universidad Nacional del Santa). Repositorio de tesis de la Universidad Nacional del Santa. http://repositorio.uns.edu.pe/handle/UNS/2625.

[4] Caballero, M y Escobedo, A. (2019). Actividad antioxidante de una bebida refrescante elaborado a partir de harina de cáscara de maracuyá (Passiflora edulis). (Título profesional, Universidad Nacional del Santa). Repositorio de tesis de la Universidad Nacional del Santa. http://repositorio.uns.edu.pe/handle/UNS/3385.

[5] Calderón, V y Noriega, V. (2017). Obtención de harina de los residuos de frutas con mayor poder antioxidante y antimicrobiano. (Título profesional, Universidad de Guayaquil). Repositorio de tesis de la Universidad de Guayaquil. http://repositorio.ug.edu.ec/handle/redug/18336.

[6] Cazares, M.J. (2011). Evaluación fisicoquímica y farinográfica de la harina de trigo (Triticum aestivum) obtenida en los pasajes de molienda de la industria "Molinos Miraflores". (Título profesional, Universidad Técnica de Ambato). Repositorio de la Universidad de Ambato. http://repositorio.uta.edu.ec/handle/ $123456789 / 834$. 
ISSNe: 2617-9156

[7] Chavez Y. (2011). Evaluación de la propiedades reológicas de masas funcionales (sin gluten). (Título profesional, Universidad Autónoma Agraria Antonio Narro). Repositorio de la Universidad Antonio Narro. http://repositorio.uaaan.mx:8080/xmlui/handle/123456 $789 / 553$.

[8] Chung J, Muro N, Ontaneda M, Palas S y Rodríguez S. (2019). Diseño de una línea de producción para la elaboración de harina a base de la cáscara de maracuyá en Quicornac S.A.C. (Proyecto educativo, Universidad de Piura). Repositorio institucional de la Universidad de Piura. Piura. https://hdl.handle.net/11042/3829.

[9] Cruz A, Guamán M, Castillo M, Glorio P y Martínez R. (2015). Fibra dietaria en subproductos de mango, maracuyá, guayaba y palmito. Revista politécnica. Vol. 36 ,

$\mathrm{N}^{\circ} 2$.

https://revistapolitecnica.epn.edu.ec/ojs2/index.php/rev ista_politecnica2/article/view/633.

[10] Decker, E.A. (1997). Phenolics: ¿prooxidants or antioxidants? Nutritional Reviews. 55 (1): 396-398. https://doi.org/10.1111/j.1753-4887.1997.tb01580.x.

[11] Elleuch, M., Bedigian, D., Roiseux, O., Besbes, S., Blecker, C., y Attia, H. (2011). Dietary fibre and fibrerich byproducts of food processing: Characterisation, technological functionality and commercial applications: A review. Food Chem., 124(2), 411

421. https://doi.org/10.1016/j.foodchem.2010.06.077.

[12] Escudero, E. y González, P. (2006). La fibra dietética. Nutr Hosp. 21(2):61-72. http://scielo.isciii.es/scielo.php?script=sci_arttext\&pid $=$ S0212-1611200600050 0007\&lng=es

[13] Grass, P; Carpenter, H. \& Anderssen, R. (2000). Modelling the developmental rheology of wheat flour dough using extension test. Journal of Cereal Science, 31 (1), 1-13. https://doi.org/10.1006/jcrs.1999.0293.

[14] Gustavsson, J., Cederberg, C., Sonesson, U. \& Emanuelsson, A. (2013). The methodology of the FAO study: global food losses and food waste - extent, causes and prevention, SIK. http://www.sik.se/archive/pdffilerkatalog/SR 857. pdf.

[15] Ivana, M. (2013). Caracterización y funcionalidad de subproductos de chía (salvia hispanica 1.) aplicación en tecnología de alimentos. (Tesis doctoral, Universidad Nacional de La Plata). Repositorio institucional de la UNLP. http://sedici.unlp.edu.ar/handle/10915/26984.

[16] Manual Megazyme. (2011). “TOTAL DIETARY FIBRE” Assay procedure, K-TDFR.

[17] Matsuura, F. (2005). Estudo do albedo de maracujá e de seu aproveitamento em barra de cereais. (Tesis doctoral, Universidade Estadual de Campinas). Repositorio institucional UNICAM http://repositorio.unicam.br/handle/ REPOSIP/254407.

[18] Miguel E. (2008). Obtención de fibra dietética a partir de piña (Ananas comosus) del cultivar cayena lisa. (Título profesional, Universidad Nacional del Centro del Perú). Repositorio UNCP. http://hdl.handle.net/20.500.12894/2632.

[19] Moreno C. (2017). Influencia de la adición de harina de cáscara de mango (Mangifera indica 1.), variedad kent y ácido ascórbico sobre las características tecnológicas del pan de molde. (Tesis doctoral, Universidad Nacional del Santa. Repositorio insitucional de la Universidad Nacional del Santa. http://repositorio.uns.edu.pe/handle/UNS/3037.

[20] Nitzke, J y Silveira, R. (2012). Procesamiento de alimentos de origen vegetal. Instituto de ciencia y tecnología de alimentos-ICTA. Ingeniería de alimentos. Universidad federal de Rio Grande, Brasil.

[21] Núñez J. (2014). Extracción, cuantificación, caracterización fisicoquímica y funcional de fibra dietaria obtenida a partir de residuos de maracuyá (Passiflora edulis f. flavicarpa Deg). Título profesional, Universidad San Francisco de Quito. Repositorio digital USFQ. http://repositorio. usfq.edu.ec/ handle/23000/2860.

[22] Palomino, L., García C., Gil. J., Rojano, B., Durango, R. (2009). Determinación del contenido de fenoles y evaluación de la actividad antioxidante de propóleos recolectados en el departa $\neg$ mento de Antioquia (Colombia). Vitae, 16(3):388-395 http://www.scielo.org.co/scielo.php?script=sci_arttext \&pid=S0121-400420 09000300013.

[23] Paucar U. (2014). Elaboración de galletas con una mezcla de harina de trigo y harina de bagazo de naranja valencia (Citrus sinensis 1.). (Título profesional, Universidad Nacional del Centro del Perú. Repositorio institucional UNCP. http://repositorio.uncp.edu.pe/handle/UNCP/1887.

[24] Polanco A. (2017). Extracción, modificación y caracterización de proteínas de amaranto. (Tesis de maestría, Universidad Veracruzana). Biblioteca digital de la Universidad Veracruzana https://www.uv.mx/mca/files/2018/01/Tesis-AnaIsabel-Polanco-Murrieta.pdf.

[25] Quintero, K. (2013). Niveles de Harina de Cáscara de Maracuyá (Passiflora edulis) en Elaboración de yogurt natural. Finca Experimental La María, MocacheEcuador 2013. (Título profesional, Universidad Técnica Estatal de Quevedo). Repositorio institucional de la 
UTEQ.

ISSNe: 2617-9156

http://repositorio.uteq.edu.ec/handle/43000/320.

[26] Ramírez, M., (2011). Desarrollo de un pan "cubilete", adicionado de semillas de Chía (Salvia hispanica L.), como fuente de fibra para niños en edad escolar. V Congreso internacional de ingeniería bioquímica México.

[27] Recalde H y Rodríguez M. (2003). Utilización de las enzimas $\square$-Amilasas y Xilanasas con Ácido LAscórbico como mejorantes de las cualidades panarias en la harina de trigo. (Título profesional, Universidad Técnica de Ambato). https://docplayer.es/111686053Universidad-tecnica-de-ambato.html.

[28] Ruiz A. (2016), Fibra dietética: definición, beneficios y métodos de cuantificación. (Título profesional, Universidad Nacional Autónoma de México). Repositorio institucional de la UNAM. https://repositorio.unam.mx/contenidos/334884.

[29] Serna, S. (2013). Química, almacenamiento e industrialización de los cereales. Editorial AGT SA. México, DF. 703 p.

[30] Terpinc P, Čeh B, Ulrih NP, Abramovič H. (2012). Studies of the correlation between antioxidant properties and the total phenolic content of different oil cake extracts. Ind Crop Prod 39(0):210-217. http://dx.doi.org/10.1016/j.ind crop. 2012.02.023.

[31] Villanueva J. 2018. Efecto de la sustitución parcial de harina de trigo (Triticum Aestivum) por harina de cáscara de maracuya (Passiflora Edulis) y harina de camote (Ipomoea Batatas) en las caracteristicas tecnologicas y sensoriales del cupcake. (Título profesional, Universidad Nacional del Santa). Repositorio institucional UNS. http://repositorio.uns.edu.pe/handle/UNS/3115.. 